\title{
Strategic Grading in the Product Acquisition Process of a Reverse Supply Chain
}

\author{
Stefan Hahler · Moritz Fleischmann
}

July 9,2015

\author{
University of Mannheim, Business School, Chair of Logistics and Supply Chain Management \\ P.O.B. 1034 62, 68131 Mannheim, Germany \\ Phone +49-621 - 181 1655, Fax +49-621- 1811653 \\ shahler@bwl.uni-mannheim.de·mfleischmann@bwl.uni-mannheim.de
}

\begin{abstract}
Most recommerce providers have moved to a quality-dependent process for the acquisition of used products. They acquire the products via websites at which product holders submit upfront quality statements and receive quality-dependent acquisition prices for their used devices.

Motivated by this development of reverse logistics practice, the aim of this paper is to analyse the product assessment process of a recommerce provider in detail. To this end, we first propose a sequential bargaining model with complete information which captures the individual behaviour of the recommerce provider and the product holder. We determine the optimal strategies of the product holder and the recommerce provider in this game. We find that the resulting strategies lead to an efficient allocation, although the recommerce provider can absorb most of the bargaining potential due to his last mover advantage.

In a second step, we relax the assumption of complete information and include uncertainty about the product holder's residual product value. We show the trade-off underlying the recommerce provider's optimal counteroffer decision and analyse the optimal strategy, using a logistic regression approach on a real-life data set of nearly 60,000 product submissions. The results reveal a significant improvement potential, compared to the currently applied strategy.
\end{abstract}




\section{Introduction}

Since the quality of a used product plays a major role in many reverse logistic environments, there are economic benefits which can be obtained by applying quality-dependent pricing strategies (Guide et al. 2003: Hahler and Fleischmann, 2013). The present paper analyses the quality assessment strategies of a recommerce provider who offers quality-dependent acquisition prices via websites to potential holders of used products. Our focus is on the individual strategic behaviour of the two players in the acquisition process when deciding upon the quality assessment.

Current practice shows a new trend in the acquisition of small electronic devices, such as mobile phones and MP3 players (see, for example, tradein.bestbuy.com and www.rebuy.de). On their websites, these so-called recommerce providers offer quality-dependent acquisition prices for used electronic devices. The owner of a used good can then select the specific model he or she wants to sell and the specific condition of the good, and subsequently receives a provisional price offer. After accepting the offer, the good is sent by mail to the recommerce provider. Upon arrival, the good is tested and compared with the product holder's description. The recommerce provider can then accept the used product and transfer the money to the product holder to close the deal. However, he also has the option to revise the offered acquisition price due to a misfit between the final grading result and the product holder's upfront grading. In that case, the product holder is offered a new, presumably lower, acquisition price. If the product holder does not accept the new offer, the product is sent back.

Actual observations of product submissions to a recommerce provider reveal that there is a significant misfit between the quality stated by the product holder and the result of the grading by the recommerce provider. A representative data set shows that the considered recommerce provider updated the provisional acquisition price for nearly $10 \%$ of the submissions. A counteroffer can on the one hand result in a complete loss of the deal, with significant costs involved, or on the other hand increase the achievable profit. It is therefore a challenging task for a recommerce provider to decide about a revised acquisition price.

In this paper, we analyse the strategic behaviour of a recommerce provider and a product holder during the acquisition process. Our main interest is to identify the product assessment strategy which optimizes the recommerce provider's achievable profit from a product submission. Thus, our explicit research question is: How should a recommerce provider act optimally in the acquisition process of individual product returns, and which factors drive his decisions? Additionally, we consider how structural changes in the acquisition process influence the behaviour of both players.

As the first step, we develop a sequential bargaining model with complete information which describes the acquisition process in detail. Using backward induction, we determine the optimal assessment strategies of 
the product holder and the recommerce provider. We also show the influence on the optimal strategy of levers like transportation cost changes and bonus payments to the product holder. In a second step, we extend our basic model by relaxing the assumption of complete information about the product holder's residual product value and analyse the recommerce provider's trade-off when deciding on a potential counteroffer. A data set consisting of nearly 60,000 product acquisitions of a recommerce provider is used to approximate the product holder's behaviour through a logistic regression approach. By comparing the benefits of an optimal counteroffer with the currently applied strategy, we find potential for improving the profitability of the recommerce provider's acquisition process.

To summarize, our paper makes the following contributions:

- We propose a modeling framework which describes the acquisition process of a recommerce provider and identify the optimal strategies of the product holder and the recommerce provider, concerning the assessment of used products. The equilibrium reveals that neither the recommerce provider nor the product holder have an incentive to properly assess the quality of the used product.

- Using this basic model, we analyse how levers like changing transportation costs or a bonus payment for a correct quality statement affect the behaviour of both players.

- We add uncertainty about the product holder's residual product value to our basic model and show the resulting trade-offs in the counteroffer decision of the recommerce provider.

- We use a data set consisting of nearly 60,000 product acquisitions of a recommerce provider for an empirical analysis of the product holder's behaviour. We derive an optimal counteroffer decision, using a logistic regression approach, in which the behaviour of the product holder is dependent on the relative price decrease of the counteroffer.

- A numerical comparison of the optimal counteroffer decision and the currently applied strategy illustrates the economic benefits of our approach. Furthermore, we find other managerial implications that improve the acquisition pricing strategy of a recommerce provider.

The remainder of this paper is organized as follows. Section 2 introduces the business of FLIP $4 N E W$ - a recommerce provider operating in Europe - which motivates our research. In Section 3, we position our work within the research literature. We then introduce our basic model with its key assumptions in Section 4 Section 5 contains the analytical results derived from our model. In Section 6 , we extend our basic model by introducing uncertainty about the product holder's residual valuation of the used product and explain our empirical estimation of the product holder's behaviour. The subsequent numerical illustration compares our 
approach with the current practice of the recommerce provider. Section 7 summarizes our main contributions and gives directions for future research. All proofs are given in the appendix.

\section{Reverse logistics at a recommerce provider}

FLIP 4 NEW offers recommerce services to individual end consumers. The business started with the launch of the website www.flip4new.de in October 2009 and has since become a prominent player in the German recommerce market. At present, the company operates in Germany and Austria. The focus of FLIP4NEW's operations was initially on the acquisition of Apple products. Today, the company repurchases a variety of used electronic goods from end consumers, ranging from used mobile phones to cameras and notebooks of different manufacturers (Taube, 2013).

FLIP $4 N E W$ acts as a broker in the second hand market. The company acquires used products from individual product holders and resells them via multiple channels to other individual consumers. Compared to self-executed direct resales, e.g. through eBay, product holders benefit from the recommerce provider's organisation of the return logistics and from the offering of fixed prices. Other recommerce providers operating with a similar business model are, e.g., wirkaufens (www.wirkaufens.de), reBuy (www.rebuy.de), Gazelle (www.gazelle.com), and Best Buy (tradein.bestbuy.com).

Figure 1 depicts the flow of a handed-in product at FLIP $4 N E W$, which is typical for the current practice in the recommerce business. First, the product holder grades the product via pre-defined quality criteria, which can be found on FLIP $4 N E W$ 's website. These quality criteria are dependent on the product category and include, e.g., the optical condition, the functionality, and the accessories included. After stating these quality features via the website, a provisional acquisition price is determined and offered. On this basis, the product holder decides whether to hand in the product and mail it to FLIP $4 N E W$. The shipment is free of charge for the product holder. After the product has arrived at FLIP $4 N E W$ 's site (Inbound), the quality of the product is checked and the actual quality of the product is determined (Grading). If the observed quality matches the quality stated by the product holder (Correct Quality), FLIP $4 N E W$ acquires the product for the agreed price. Dependent on the product category and the specific quality of the product, FLIP $4 N E W$ executes process steps, such as, e.g., cleaning and deletion of personal data (Processing). Subsequently, the product moves into the storage area (Storage). If the product holder's stated product quality deviates from the recommerce provider's assessment (Wrong Quality), FLIP $4 N E W$ has to choose between issuing an adjusted counteroffer or buying the product for the original price despite the quality deviation. In case of a counteroffer, the product holder receives a new purchasing offer from FLIP $4 N E W$ via email. During the time that FLIP $4 N E W$ is waiting for the response on the counteroffer, the used product is stored in an 


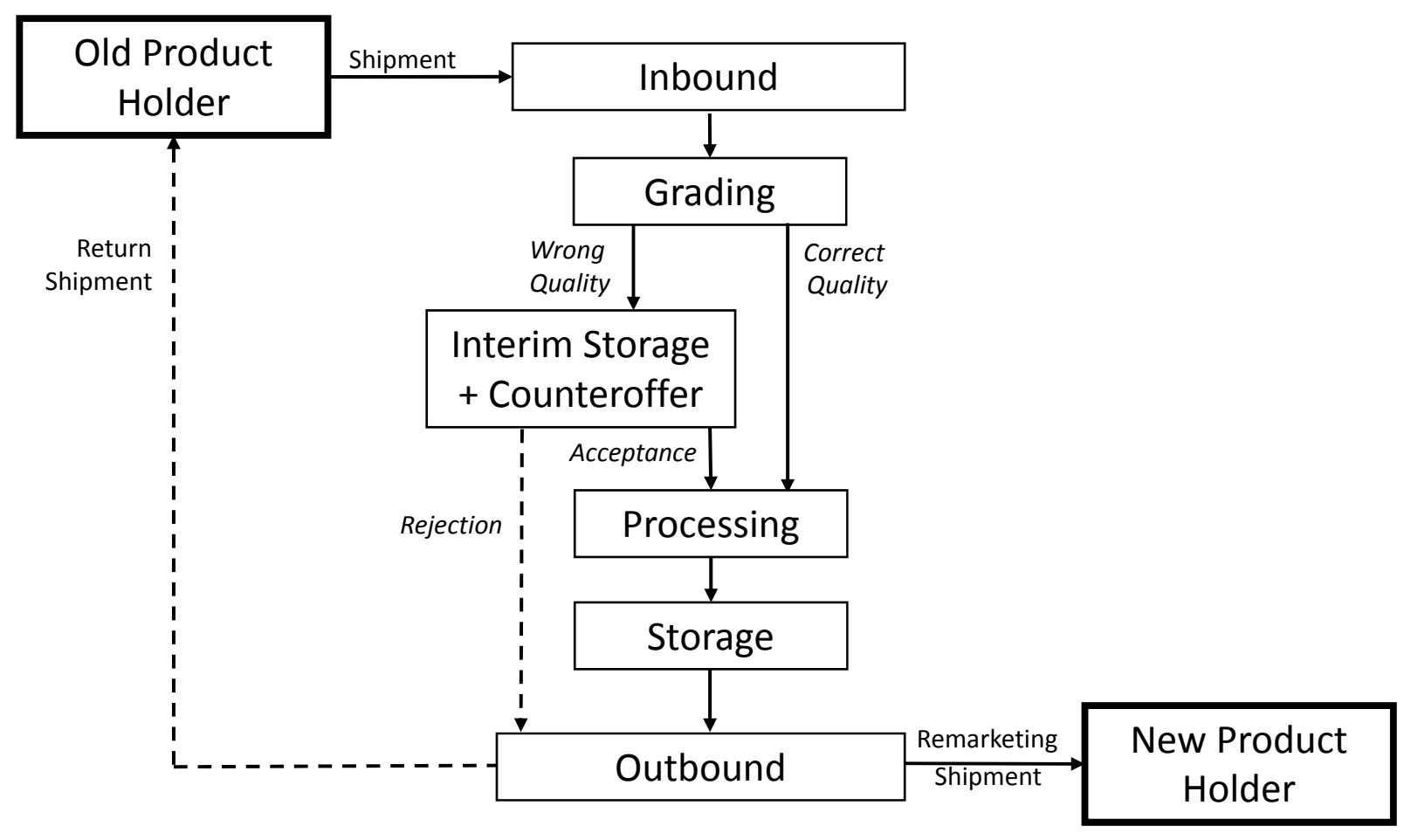

Figure 1: A typical product flow at a recommerce provider like FLIP $4 N E W$

interim storage area. If the product holder accepts the new offer, the product is acquired by the company and moves via a processing step into the storage area. In case of a rejection of the new price offer by the product holder, FLIP $4 N E W$ sends the product back to the holder (Outbound). FLIP $4 N E W$ then bears all transportation costs.

For the stored and acquired used products, FLIP $4 N E W$ starts the remarketing directly. An important redistribution channel to the consumer market is the 'buy it now'-option of its cooperating partner eBay. Most of the acquired products are sold via this channel. FLIP $4 N E W$ generates a separate offer for every product with photos and a detailed description of its quality. When the used product is resold FLIP4NEW ships it to the new owner.

The research literature commonly structures the processes of a reverse supply chain according to three stages, namely back end (remanufactured products market development), engine (remanufacturing operational issues), and front end (product returns management) (Guide and Jayaraman, 2000, Guide and Wassenhove, 2009). The above description covers this scope for the case of FLIP $4 N E W$. Our analysis in this paper addresses the front-end acquisition process and specifically the managerial challenges arising from the upfront quality grading process. One major issue is the decision about the acceptance or rejection of the returned products, given the product holders' quality statements. On the one hand, a counteroffer can result in a 
complete loss of the acquisition, with significant costs involved for the recommerce provider. On the other hand, the counteroffer potentially allows the recommerce provider to increase his margin by negotiating a lower price. Thus, it is a challenging task for a recommerce provider to decide on an update of the acquisition price.

FLIP $4 N E W$ currently manages this decision by issuing a counteroffer based on the relative difference between the price for the true quality and the stated quality. If this difference exceeds a certain threshold the company will make a counteroffer; otherwise it accepts the stated quality and pays the original acquisition price. Despite this generous acceptance policy, FLIP 4 NEW issues counteroffers for nearly $10 \%$ of the submitted products. This shows that quality mismatches are a significant issue, which requires a systematic response. The goal of this paper is to address this issue by investigating the decisions of a recommerce provider like FLIP 4 NEW and a product holder in the used-product acquisition process.

\section{Literature}

Souza (2013) gives a broad and recent review of the field of closed-loop supply chains and highlights further research opportunities. He emphasizes the need for practice-driven empirical research, especially on the acquisition and collection process, and on consumer behaviour in this field. The same recommendations for future approaches are given by Guide and Wassenhove (2009).

The problem setting considered here concerns two main processes of a reverse supply chain - acquisition and grading. Several issues in reverse logistics with a strong focus on these two processes have been analysed in the literature. We refer to Fleischmann et al. (2010) for a broad overview. In the following, we indicate the research papers most closely related to our study.

The research field of profit-oriented used-product acquisition management was initiated by Guide and Jayaraman (2000), and Guide and Wassenhove (2001). Prior work in the field of reverse logistics commonly assumed an exogenous return process. Furthermore, Guide et al. (2003) introduced quality-dependent acquisition prices, which allow managing the quantity and quality of the returned products. Other important papers following their approach include Ray et al. (2005), who consider a continuous quality differentiation rather than discrete quality classes, and Karakayali et al. (2007), who consider different reverse channel structures of an OEM and determine optimal acquisition prices for remanufactured parts. Our work is strongly related to this research stream as we consider a profit-oriented recommerce provider who offers quality-dependent prices for used products to individual product holders. Nevertheless, our focus is not on the pricing decision itself. Instead we consider the strategic quality statements during the acquisition process, both from the recommerce provider and the product holder. These quality statements indirectly 
determine the final acquisition price.

For the research literature on the used-product grading process, we adopt the classification of Fleischmann et al. (2010), dividing this field into two streams. The first stream considers the value of the grading information for the subsequent processing of the returned products. Souza et al. (2002) and Ketzenberg et al. (2003) were the first to consider the grading process in this context. Ketzenberg et al. (2003) find that the availability of grading information generally improves flow times in the remanufacturing system. Similar studies analysing the value of the grading information by comparing a remanufacturing system with and without an upfront grading process include Guide et al. (2005), Aras et al. (2004), and Zikopoulos and Tagaras (2008). In our setting, the holder of the used product performs an upfront quality grading. However, the focus of our work is not on the value of this grading information for the operational performance of the remanufacturing system, but rather on its impact on the recommerce provider's strategic product assessment. Thus, we contribute to this stream by considering how the upfront quality grading influences the acquisition process.

The second literature stream on used-product grading compares multiple options for the grading process. Two important studies in this stream which are strongly related to our work are Guide et al. (2006) and Blackburn et al. (2004). They evaluate different grading locations in the return process. One major result is that a less time-consuming decentralized grading set-up can be more beneficial than a cost-efficient slower one, especially for used products with a high marginal value of time. These high marginal values of time are common, e.g., for most returns in the electronic goods industry. In our problem setting, the focus of a recommerce provider is on electronic goods from end consumers. Thus, we also incorporate the time value loss in our analysis of the acquisition process and observe its impact on the grading strategy of the recommerce provider and the product holder. Additionally, we contribute to this stream by analysing different payment structures in the acquisition process and showing the impact of these levers on the outcome of the acquisition process.

To our knowledge, there is no work in the literature which takes into account the upfront quality grading in the above described acquisition process in the recommerce business and which analyses the resulting strategic interaction between the recommerce provider and the product holder. Our aim is to fill this research gap, which has been identified by Hahler and Fleischmann (2013). By doing so, we contribute to the literature as we identify the counteroffer decision in the acquisition process of a recommerce provider as a new business decision in the field of reverse logistics. Furthermore, our work connects the research streams on the acquisition and grading processes. Additionally, a close collaboration with a recommerce provider during this project yielded new insights into this fast developing industry. Thus, our research approach addresses the needs identified by Souza (2013) and Guide and Wassenhove (2009) as we consider a novel 
practice-driven problem setting and provide empirical results from an actual data set from the recommerce industry.

There are two recent research papers which are related to our problem setting. The first one is the aforementioned work by Hahler and Fleischmann (2013). The paper compares two collection system configurations from the reverse logistics industry. One is a decentralised system in which the grading is done close to the product holder, whereas the other system has a centralised grading process. As a centralised collector has almost the same business model as a recommerce provider, most of the results concerning the collection network design and corresponding grading strategy can be adapted to our case. However, our focus in this paper is on the strategic interaction between the players involved in the acquisition process. This interaction is not included in the analysis of Hahler and Fleischmann (2013).

The second closely related research paper is Gönsch (2014). The paper compares two acquisition pricing strategies. In the first strategy, the manufacturer offers fixed acquisition prices. In the second strategy, the collector bargains with the product holder. Furthermore, Gönsch (2014) shows how competition from a thirdparty remanufacturer influences the results, compared with a monopolistic setting. The main difference from our work is that Gönsch (2014) considers only one time period, and thus does not focus on the negotiation process itself. He instead divides the total surplus of the product submission by a factor describing the relative bargaining power of each player. The aim of our work is to analyse the business decisions of a recommerce provider in the acquisition process from a practice perspective. We develop a sequential game for capturing the corresponding interactions in detail.

\section{Basic model}

Following the setting described in Section 2, we consider a single product submission of an individual product holder to a recommerce provider and analyse the decisions of both players during the acquisition process. We model this setting as a sequential game. The product holder (he) decides on submitting his used product for a specific price and is faced with a possible counteroffer at the end of the game. The recommerce provider (she) either accepts the provisional acquisition price or offers a new price for the used product. The interaction during the acquisition process is shown in Figure 2. The illustrated sequential game indicates the moves of the recommerce provider and the product holder. The corresponding payoffs for the product holder and the recommerce provider are indicated in brackets at the four possible endpoints of the game.

In the following, we present our model assumptions and provide a short discussion. Then, we describe the single steps of the game in detail. The notation is summarized in Table 1. 


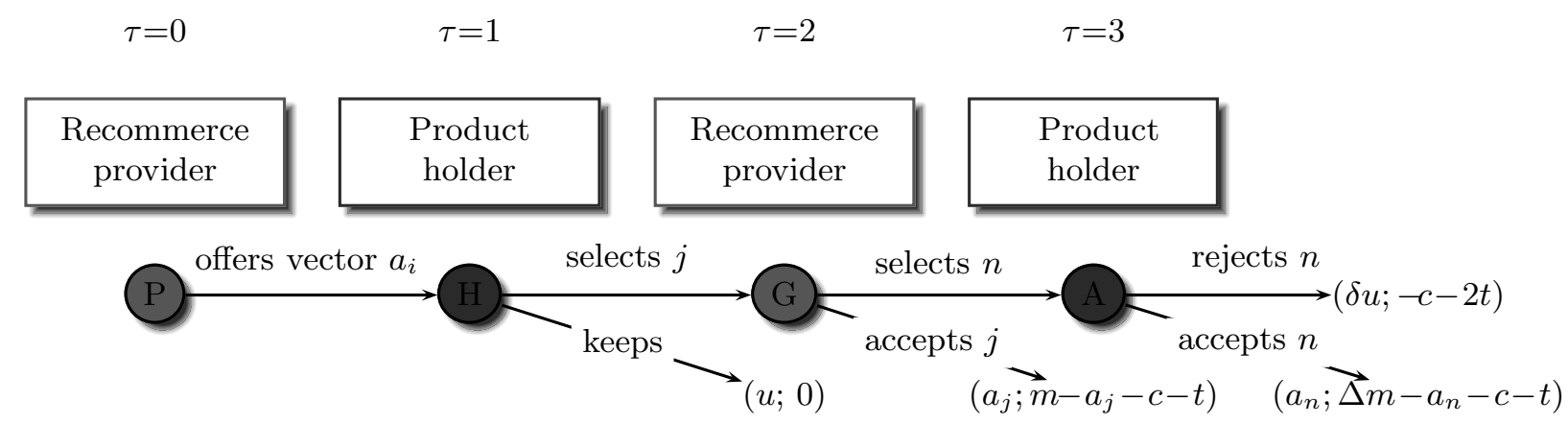

Figure 2: Acquisition process of a recommerce provider

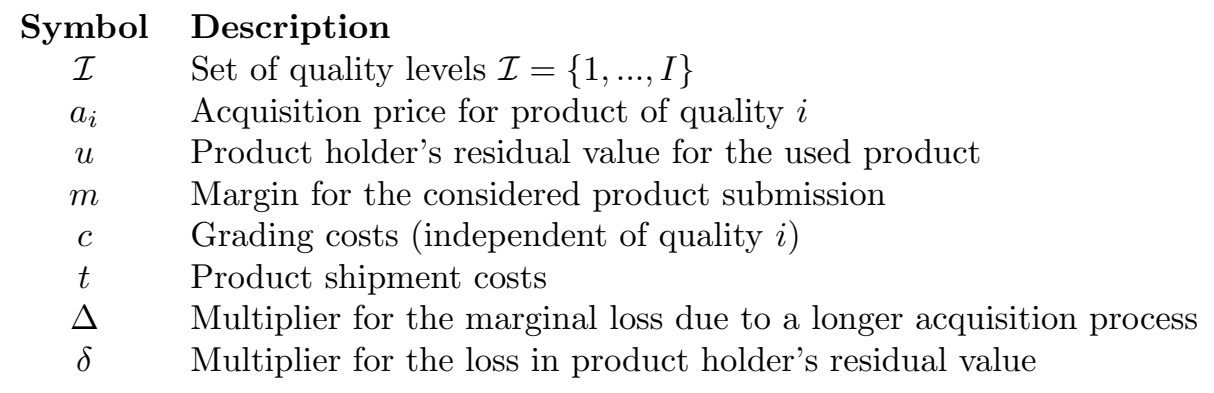

Table 1: Notation

\subsection{Assumptions}

1. Assumption: The recommerce provider distinguishes I different quality classes and offers a vector of quality-dependent acquisition prices $a_{i}$ for $i \in \mathcal{I}$.

The recommerce provider specifies the individual quality classes $i$ and the corresponding prices $a_{i}$. The pricing flexibility of the recommerce provider is strongly limited as she has to set the prices in a competitive environment; the level of quality differentiation is restricted for reasons of customer usability. Thus, we assume that the prices and quality classes are exogenous in the sequential game and are not adjusted for an individual customer. In Section 6, we derive recommendations for defining the price differences between two quality classes, based on the analysis of the practice data set.

2. Assumption: The recommerce provider achieves a reselling price for the considered product submission on the secondary market which determines the achievable margin $m$.

As in Hahler and Fleischmann (2013), we focus on the purchase of used products by the recommerce provider and assume the market price for a specific output quality to be fixed. Consequently, the recommerce provider is a price taker on the resell market. We consider a margin $(m)$ which is the market price reduced by the costs which occur for any product that is resold. These costs are, e.g., the processing costs for cleaning and data deletion, costs for remarketing, and shipment costs. Thus, the disposition decision of the recommerce 
provider is defined exogenously by the specific quality class of the used product.

3. Assumption: The recommerce provider incurs grading costs $c$ and shipment costs $t$.

The process for determining the true quality of the used product results in costs $c$ which are independent of the quality. For the shipment of the used product from the product holder to the site of the recommerce provider, the recommerce provider pays transportation costs $t$. In the base case, we assume additionally that the recommerce provider pays the return shipment costs of the product if the product holder does not accept a given counteroffer.

4. Assumption: We consider an individual product holder who decides about using the service of the recommerce provider. This product holder attributes a residual value u to the product.

Because the used product can still be used, e.g., as a replacement device, the used product has a residual value for the product holder.

5. Assumption: If the acquisition process is delayed due to a counteroffer of the recommerce provider the achievable margin $m$ in the payoff of the recommerce provider is discounted by $\Delta \in[0 ; 1]$. Analogously, the holder's residual value of the used product is discounted by $\delta \in[0 ; 1]$.

There are product submissions for which the acquisition process takes four weeks, due to long response times for the counteroffers. As noted in Section 2 the considered products lose value during that time period. This value loss is captured by the multiplier $\Delta$.

We discount the product holder's residual value of the used product by $\delta$ because the product is not available for use during the transaction process. As described above, this can take up to four weeks. Furthermore, the product holder has an additional handling effort to get the product back.

6. Assumption: In the sequential game, the product holder and the recommerce provider have complete information and act rationally.

In what follows, we will describe the acquisition process of the recommerce provider as a finite sequential game. We assume that each player has complete information. Thus, each player is aware of the rival's prior moves and knows the full history of the game thus far. In Section 6 , we relax the complete information assumption and introduce uncertainty about the product holder's residual value $u$.

\subsection{Description of the sequential game}

The game unfolds in four steps, as shown in Figure 2. $\tau=0$ : The recommerce provider announces $I$ discrete quality classes and their corresponding acquisition prices $a_{i}$.

$\tau=1:$ The product holder assesses the quality of his used product and receives an offer via the website of 
the recommerce provider for the selected quality class (we use $j$ to describe the selected quality class after the upfront quality grading of the product holder). At this point, the product holder makes an individual decision to hand in the product, based on the offered acquisition price $a_{j}$. If he accepts the offer he sends the product to the recommerce provider. Otherwise, the product holder keeps the used product and the game terminates. In this case, the product holder still has the residual value $u$ of the product. As the product holder can manipulate the stated quality in his favour the price $a_{j}$ can be considered as an offer for sale by the product holder to the recommerce provider.

$\tau=2$ : The product quality will be determined by the recommerce provider after the shipment has arrived. The result of the grading process is denoted by $r$ (true quality), which may or may not be equal to the quality stated by the product holder, $j$. At this point, the recommerce provider has to decide whether to accept the initial acquisition price or to offer an adjusted one. If she accepts the used product for the stated quality the product holder gets the acquisition price $a_{j}$ and the recommerce provider achieves $m-a_{j}-c-t$. If the recommerce provider chooses to update the acquisition price the product holder receives a counteroffer for handing in the used product. We use $n$ to denote the new offered quality, which does not need to coincide with the observed (graded) quality $r$.

$\tau=3$ : The last step is the product holder's decision whether or not to accept the updated acquisition price $a_{n}$. He can accept the new offer, which results in payoffs of $a_{n}$ for the product holder and $\Delta m-a_{n}-c-t$ for the recommerce provider. We do not discount the delayed payment of the acquisition price $a_{n}$ as we assume that the cost of capital is negligible in comparison with the loss in value of the used product.

If the product holder rejects the new offer, the recommerce provider returns the product to him. This results in payoffs of $-c-2 t$ for the recommerce provider because she bears the shipment costs $t$ for returning the product. The payoff for the product holder is $\delta u$.

\section{Analysis}

We use the described basic model to analyse the individual decisions of the product holder and the recommerce provider in the acquisition process. We use backward induction to determine each player's optimal strategy and find the equilibrium of the current grading process. Furthermore, we analyse the influence of two potential changes in the payment schemes on the optimal strategies of both players. 


\subsection{Optimal bargaining strategies}

\subsubsection{Product holder's acceptance decision $(\tau=3)$}

Assume that the product holder handed in the used product and was offered an updated acquisition price $a_{n}$ by the recommerce provider. At this moment, the product holder's decision is to accept or reject the counteroffer. A rational product holder will accept the counteroffer $a_{n}$ if it is larger than the discounted residual value $\left(a_{n} \geq \delta u\right)$. Otherwise $\left(a_{n}<\delta u\right)$, it is optimal for the product holder to reject the new offer and have the product returned.

\subsubsection{Recommerce provider's acceptance and counteroffer decision $(\tau=2)$}

At $\tau=2$, the recommerce provider decides whether to accept or reject the provisional acquisition price $a_{j}$. In the latter case, she also decides on the counteroffer $a_{n}$ to make to the product holder. As this decision consists of two steps, we begin with the selection of the optimal counteroffer $a_{n}$.

1) The optimal counteroffer $a_{n}$ depends on the payoffs for the recommerce provider in the last step of the sequential game. The recommerce provider compares the payoff for a rejection of the counteroffer $(-c-2 t)$ with the payoff of an acceptance $\left(\Delta m-a_{n}-c-t\right)$. As the recommerce provider anticipates that the product holder will accept the counteroffer $a_{n}$ if it is greater than or equal to the discounted residual value $\delta u$, she has to offer at least $\delta u$ to incentivize the product holder to accept the new offer. Thus, we define $a_{n}(\delta u)$ as the smallest $a_{n}$ which satisfies $a_{n} \geq \delta u$. Using this notation, the optimal decision of the recommerce provider is $a_{n}(\delta u)$ if the following holds:

$$
\begin{aligned}
\Delta m-a_{n}(\delta u)-c-t & \geq-c-2 t \\
\Leftrightarrow \quad a_{n}(\delta u) & \leq \Delta m+t .
\end{aligned}
$$

Otherwise, it is optimal to offer a price which is lower than the residual value, knowing that this counteroffer will be rejected by the product holder.

2) In a second step, the recommerce provider compares the payoff from the optimal counteroffer decision with the payoff from an immediate acceptance of $a_{j}$.

a) If the optimal counteroffer decision is $a_{n}(\delta u)$ the recommerce provider accepts $a_{j}$ if the following 
holds:

$$
\begin{aligned}
& m-a_{j}-c-t & \geq \Delta m-a_{n}(\delta u)-c-t \\
\Leftrightarrow & (1-\Delta) m & \geq a_{j}-a_{n}(\delta u) .
\end{aligned}
$$

Linking Inequality (1) with (2) results in the following condition for this case:

$$
a_{j} \leq a_{n}(\delta u)+(1-\Delta) m \leq m+t .
$$

b) If the optimal counteroffer is lower than the residual value (Inequality (1) is not satisfied), the recommerce provider accepts $a_{j}$ if the following holds:

$$
\begin{aligned}
& m-a_{j}-c-t \geq-c-2 t \\
& \Leftrightarrow \quad m+t \geq a_{j} .
\end{aligned}
$$

Linking again both conditions results in the following inequalities for this case:

$$
a_{j} \leq m+t \leq a_{n}(\delta u)+(1-\Delta) m .
$$

Summing up, the recommerce provider's optimal decision is to accept $a_{j}$ if:

$$
a_{j} \leq \min \left(a_{n}(\delta u)+(1-\Delta) m, m+t\right) .
$$

\subsubsection{Product holder's hand-in decision $(\tau=1)$}

The product holder's first decision in the sequential game is whether to hand in or to keep the used product. In the former case, he also has to decide which quality $j$ to state for the submission. Thus, the product holder can keep the product with its residual value $u$ or can achieve one of the three other possible end points of the sequential game with their respective payoffs, depending on the specific quality selection. In the following, we consider the conditions that have to be fulfilled to obtain each of these payoffs:

1. $a_{j}$ : As we have identified above, the recommerce provider will accept the provisional acquisition price $a_{j}$ if it satisfies Inequality (6), i.e. $a_{j} \leq \min \left(a_{n}(\delta u)+(1-\Delta) m, m+t\right)$.

2. $a_{n}(\delta u)$ : The product holder can trigger the counteroffer $a_{n}(\delta u)$ if the selected quality $j$ leads to a provisional acquisition price which satisfies $a_{j}>\min \left(a_{n}(\delta u)+(1-\Delta) m, m+t\right)$ and $a_{n}(\delta u)+(1-\Delta) m \leq$ 
$m+t$.

3. $\delta u$ : The product holder obtains the discounted residual value $\delta u$ if the selected quality $j$ leads to a provisional acquisition price which satisfies $a_{j}>\min \left(a_{n}(\delta u)+(1-\Delta) m, m+t\right)$ and $a_{n}(\delta u)+(1-\Delta) m>$ $m+t$. The corresponding counteroffer of the recommerce provider will be lower than the discounted residual value of the product holder, and thus the offer will be rejected.

4. $u$ : The product holder obtains the residual value $u$ when deciding to keep the product at the beginning of the sequential game.

We observe that the third end point $(\delta u)$ is by definition lower than the residual value at the beginning of the game $(u)$. Thus, it is never optimal for the product holder to arrive at this outcome. Furthermore, we assume in the following that the recommerce provider's quality differentiation is sufficiently granular so that her optimal counteroffer is always below the product holder's residual value (i.e. $\left.a_{n}(\delta u) \leq u\right)$. This implies that the second payoff $\left(a_{n}(\delta u)\right)$ is also not desirable for the product holder.

The first payoff $a_{j}$ will be realized if the recommerce provider accepts the provisional acquisition price immediately. If there exist more than one price $a_{j}$ which fulfills this condition then the product holder's optimal acquisition price $a^{*}$ will be the largest one of these, i.e.

$$
a^{*}=\max \left\{a_{j} \mid a_{j} \leq \min \left(a_{n}(\delta u)+(1-\Delta) m, m+t\right)\right\}
$$

Consequently, the product holder's hand-in decision depends on comparing the highest achievable payoff $a^{*}$ with the residual value $u$. If the optimal provisional acquisition price $a^{*}$ is higher than the residual value of the product $u$ he will hand in the product, otherwise he will keep it. We add this property to Equation (7), which leads to the optimal provisional acquisition price $a^{*}$ :

$$
a^{*}=\max \left\{a_{j} \mid u \leq a_{j} \leq \min \left(a_{n}(\delta u)+(1-\Delta) m, m+t\right)\right\}
$$

If this set is empty it is optimal for the product holder to keep the used product.

Consider Equation 8 for the case $a_{n}(\delta u)+(1-\Delta) m>m+t$. Then handing in the product is optimal if the following two conditions are satisfied simultaneously:

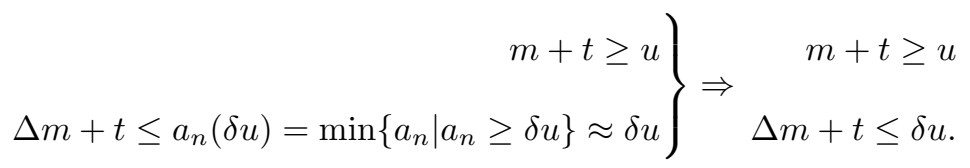


However, these conditions can only be satisfied simultaneously if the marginal loss over time of the used product $(\Delta)$ is much larger than the discounting of the residual value $(\delta)$, or if the quality differentiation granularity is very low. As these are not the case for our acquisition setting, we neglect this theoretical solution of the game in the following and focus in Equation (8) on the case $a_{n}(\delta u)+(1-\Delta) m>m+t$. Thus, the optimal strategy of the product holder is to select the stated quality $j$ with the highest $a_{j}$ as follows:

$$
a^{*}=\max \left\{a_{j} \mid u \leq a_{j} \leq a_{n}(\delta u)+(1-\Delta) m\right\}
$$

If there is no quality class $j$ which satisfies these conditions, the optimal strategy of the product holder is to keep the used product. In the following, we take a closer look at this equilibrium solution.

\subsection{Analysis of the equilibrium solution}

When the used product is handed in, the optimal strategies of the product holder and the recommerce provider reveal an interesting equilibrium. The product holder will state the product quality $j$ so as to receive the maximum acquisition price $a^{*}$ which the recommerce provider is still willing to accept. The price $a^{*}$ is limited as the recommerce provider has the advantage of the last offer in the acquisition process. Thus, she always has the possibility to downgrade the product and hence can minimize the acquisition price. In finite sequential games, this property is called the last-mover advantage.

Rearranging the optimal quality selection of the product holder described in Equation (9) yields the following:

$$
\underbrace{a^{*}-a_{n}(\delta u)}_{\begin{array}{c}
\text { Additional gain } \\
\text { by downgrading }
\end{array}}<\underbrace{(1-\Delta) m .}_{\begin{array}{c}
\text { Loss of market } \\
\text { value }
\end{array}}
$$

We can interpret this inequality from the perspective of the recommerce provider's trade-off in the counteroffer decision. The term on the lefthand side describes her acquisition cost savings when she decides to downgrade the product. The term on the righthand side defines the value loss of the used product caused by prolonging the acquisition process. Inequality 10 shows that, given the product holder's optimal strategy, the recommerce provider can further downplay the product value by no more than the loss in market value. Thus, a counteroffer is not profitable.

We can make two additional observations from Inequality 107 . We denote the 'correct' acquisition price by $a_{r}$, i.e. the price for the actual product quality $r$. Interestingly, Equation (9) is independent of $a_{r}$. Depending on the equilibrium solution for the optimal provisional acquisition price $a^{*}$, the 'correct' acquisition price 
$a_{r}$ can be higher or lower than $a^{*}$. If $a^{*}<a_{r}$, the equilibrium solution improves the outcome for the recommerce provider as she can reduce the acquisition price. If $a^{*}>a_{r}$, the product holder obtains a higher gain. Depending on the relation between $a^{*}$ and $a_{r}$, Inequality 10 shows in which range the product holder or the recommerce provider can over- or understate the acquisition price. We call this observation the 'incentive problem in quality grading' because the equilibrium solution reveals that there is no incentive for either player to make an honest quality statement.

Comparing the solution of the optimal strategies in the bargaining game with the other possible solutions reveals that the summed-up payoffs in the equilibrium are higher than in any other later outcome of the sequential game. This holds because the product faces a significant loss of market value over time. Furthermore, we observe by a comparison that no player can improve his or her payoff without reducing the payoff of the other player. Thus, the equilibrium solution is efficient (Fudenberg and Tirole, 1991).

The total potential profit in the game is $m-c-t-u$. A closer look at the profit allocation between the two players shows that the recommerce provider gains most of the profit in the game, due to her lastmover advantage. The equilibrium shows that the product holder gains $(1-\Delta) m+a_{n}(\delta u)-u$ whereas the recommerce provider obtains $\Delta m-a_{n}(\delta u)-c-t$. Approximating $a_{n}(\delta u) \approx \delta u$ and $\Delta \approx \delta$, we can simplify the above allocation to $(1-\Delta)(m-u)$ for the product holder and $\Delta(m-u)-c-t$ for the recommerce provider. Thus, the multiplier of the value loss over time $(\Delta)$ defines how the profit is shared between the two players. As, in general, $(1-\Delta) \ll \Delta$ (and $(1-\delta) \ll \delta)$, the recommerce provider gains most of the potential profit. However, the recommerce provider must also bear the costs $(c+t)$. Inequality (10) explains this allocation. Due to the last-mover advantage, the recommerce provider can reduce the product holder's payoff at the end of the game to the user's discounted residual value. Additionally, the product holder anticipates that the recommerce provider wants to avoid a payoff with a discounted margin in the last period of the game. Thus, the product holder can overstate the discounted residual value by at most the amount of the product's time value loss $(1-\Delta)$.

\subsection{Effects of levers on the equilibrium solution}

\section{Transportation cost shift towards product holder}

The new EU directive on consumer rights (European Commission, 2013) gives e-commerce providers more freedom for operating their businesses. Especially, regulations concerning the burden of the transportation costs have been relaxed. Motivated by this current situation, we consider in the following a structural change in the acquisition process and evaluate the effect on the optimal strategies in the sequential game. We shift the transportation cost $t$ towards the product holder when rejecting the counteroffer of the recommerce 
$\tau=0 \quad \tau=1 \quad \tau=2 \quad \tau=3$

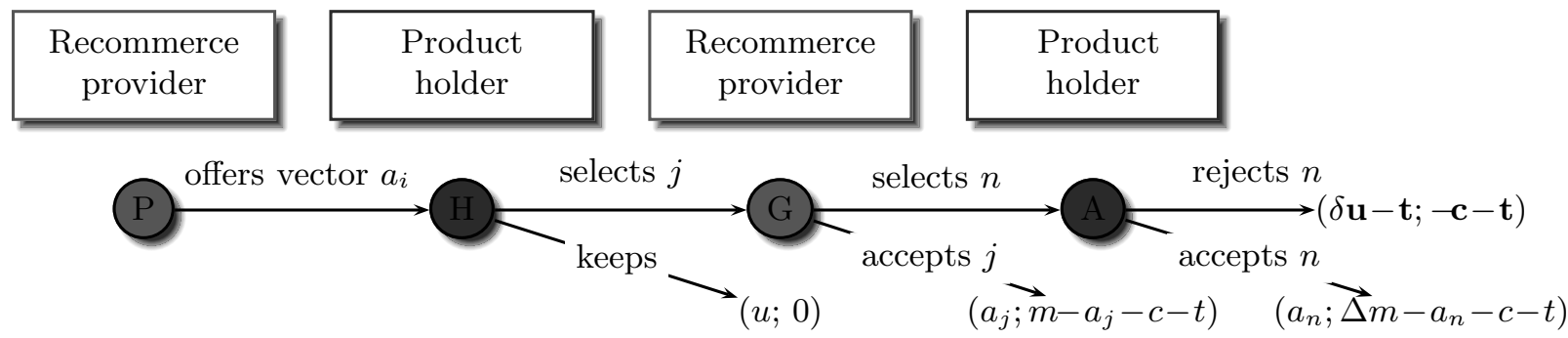

Figure 3: Acquisition process with a shift of the return shipment costs towards the product holder

$$
\tau=0 \quad \tau=1 \quad \tau=2 \quad \tau=3
$$

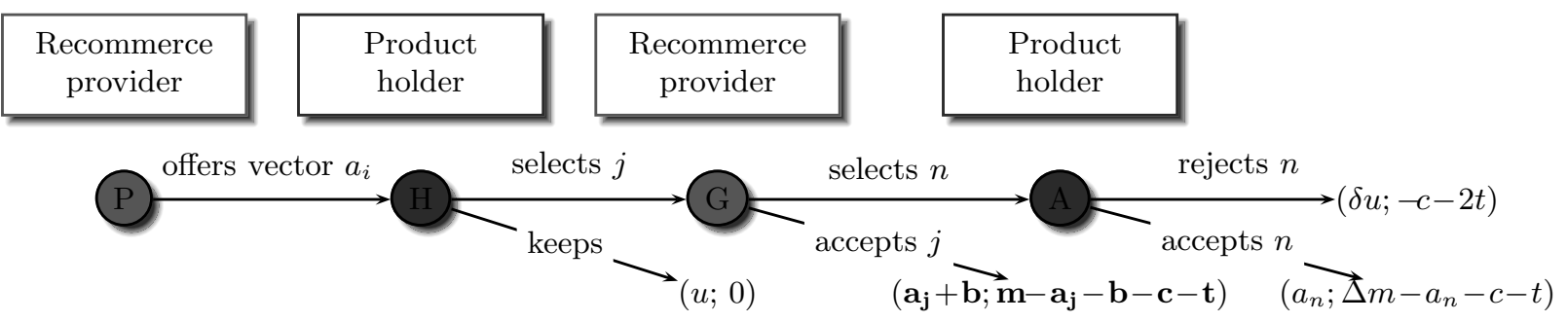

Figure 4: Acquisition process with bonus payments $b$ for correct quality assessment

provider. Figure 3 shows the acquisition process with the adjusted payoffs.

The following equation shows the new equilibrium solution when resolving the adjusted sequential game in Figure 3

$$
a^{*}=\max \left\{a_{j} \mid u \leq a_{j} \leq a_{n}(\delta u)-t+(1-\Delta) m\right\}
$$

We see that the discounted residual value of the product holder will be reduced by the shipment costs and, thus, the recommerce provider can obtain a higher gain by downgrading the product. As the product holder anticipates this behaviour when deciding to hand in the used product, the initial price offer $\left(a_{j}\right)$ has to be reduced. Evaluating Equation (11) shows the new profit share of $(1-\Delta) m+a_{n}(\delta u)-u-t$ for the product holder and $\Delta m-a_{n}(\delta u)-c$ for the recommerce provider. A comparison with the base case shows that the transportation costs $t$ are in effect shifted towards the product holder.

\section{Bonus payment for correct quality assessment}

Current product submissions to FLIP 4 NEW show that a significant number of product holders give wrong quality statements for their used products. As potential remedy, we evaluate in the following a bonus payment $b$ from the recommerce provider for the product holder in the case that the recommerce provider accepts the provisional acquisition price $a_{j}$ directly. See Figure 4 for the illustration of the changes. 
The additional bonus payment $b$ is paid if the quality statement of the product holder is accepted. The following equation shows the new equilibrium solution for the adjusted sequential game in Figure 4

$$
a^{*}=\max \left\{a_{j} \mid u \leq a_{j} \leq a_{n}(\delta u)-b+(1-\Delta) m\right\}
$$

As Equation 12 has the same structure as Equation (11), one might expect a similar effect of the bonus payment as that of the transportation cost shift. The additional acquisition cost reduction upon downgrading by the recommerce provider increases in relation to the loss of market value. The major difference between this lever and the above transportation cost shift is that the product holder receives the bonus payment $b$. Thus, reassessing the profit share reveals the same allocation as in the base case. The product holder still gains $(1-\Delta) m+a_{n}(\delta u)-u$ and the recommerce provider $\Delta m-a_{n}(\delta u)-c-t$. This shows that an additional bonus payment has no influence on the profit allocation of this solution of the game. This result can be explained by the fact that the equilibrium of an immediate offer acceptance by the recommerce provider is already achieved in Section 5.1. The additional incentive of the bonus payment will be factored in by the product holder, and thus has no effect.

\section{Uncertain residual product valuation}

A key factor which drives the results of the sequential game analysed in the previous section is the assumption of complete information. Specifically, the recommerce provider's knowledge of the product holder's residual value gives her the last mover advantage when making a counteroffer. We relax this assumption in the following and assess the resulting model empirically.

To this end, we extend our basic model and incorporate uncertainty about the residual value of the product holder. Then we introduce the used product and consumer data of FLIP $4 N E W$ and propose an approach for estimating the individual behaviour of the product holder. We assume that the counteroffer acceptance decision is dependent on the new counteroffer $a_{n}$, and we use the historical consumer data for a logistic regression to derive the acceptance probability of a specific counteroffer.

By incorporating this behaviour into the decision model, we can derive the optimal counteroffer decision of the recommerce provider under uncertainty. At the end of this section, we compare the optimal strategies with the current product assessment strategy of FLIP $4 N E W$. 


\subsection{Extension of the basic model's counteroffer decision}

We assume in the following that the recommerce provider has no exact information about the discounted residual value $\delta u$ of the individual product holder who handed in the used device. We incorporate this uncertainty about the discounted residual value with the help of a probability distribution. As the results of the previous section have shown, the product holder's final decision depends on the relation of the counteroffer $a_{n}$ to the discounted residual value $\delta u$. If $\delta u<a_{n}$, the product holder accepts, otherwise he rejects the counteroffer. Given the uncertain value of $\delta u$, we define the probability for the acceptance of a counteroffer as $\operatorname{Prob}\left(\delta u<a_{n}\right)=: P\left(a_{n}\right)$. Consequently, the probability for the acceptance of a counteroffer is increasing in $a_{n}$.

For this new setting, we reconsider the counteroffer decision $a_{n}$ of the recommerce provider. We extend the basic model by the probability function $P\left(a_{n}\right)$.1 We use this function to derive weights for the possible payoff realizations in response to a counteroffer and get the following expression for the recommerce provider's expected profit function:

$$
E\left[\Pi^{c}\left(a_{n}\right)\right]=P\left(a_{n}\right)\left(\Delta m-a_{n}-c-t\right)+\left(1-P\left(a_{n}\right)\right)(-c-2 t) .
$$

Equation 13 illustrates the recommerce provider's trade-off between the risk of losing the deal with the product holder, weighted with the probability $\left(1-P\left(a_{n}\right)\right)$, and obtaining the desired price reduction, with probability $P\left(a_{n}\right)$.

\subsection{Empirical estimation of individual product holder behaviour}

We now introduce the data set which we used to estimate the individual product holder behaviour. The data set consists of 60,000 used-product submissions to FLIP 4 NEW in the period from June 2011 to November 2012. The used products are divided into six different product categories: Mobile Phones (Phones), Tablets E3 E-Book Readers (Tablets), MacBooks (MacBooks), Macs \& Accessories (Macs), Notebooks \&3 Netbooks (NoteNet), and Cameras (Cameras).

As we aim to approximate the individual behaviour of the product holders when confronted with a counteroffer, we consider the submissions for which FLIP $4 N E W$ made a counteroffer. The data set contains counteroffers for 5,958 submissions of which 2,030 (34.1\%) were rejected by the product holders. The data for two illustrative examples are shown in Table 2.

\footnotetext{
${ }^{1}$ We provide a detailed explanation of how the probability function can be estimated from a data set of product submissions in the next subsection.
} 


\begin{tabular}{lllcccccc} 
Released & Category & Type & Arrived & $a_{j}$ & $a_{n}$ & Sold & $m$ & $Y$ \\
\hline $03 / 06 / 11$ & Phones & iPh. 4 16GB & $06 / 06 / 11$ & 358 & 304 & $23 / 06 / 11$ & 379.6 & 1 \\
$06 / 06 / 11$ & Phones & iPh. 2 8GB & $17 / 06 / 11$ & 110 & 77 & - & - & 0
\end{tabular}

Table 2: Data for two examples of product submissions

The last column of the table indicates whether the counteroffer was accepted $(Y=1)$ or rejected $(Y=0)$. We use the indicator $Y$ as the dependent variable in our subsequent analysis.

As the product holder's acceptance of the counteroffer is a strict "yes" or "no" decision, we conducted a binary logistic regression analysis for predicting this decision, for a given counteroffer Hosmer Jr et al. 2013). We used the software IBM SPSS Statistics to carry out the analysis, based on the data set described above.

The prediction of the product holder's behaviour is based on the counteroffer $a_{n}$. As the different product categories contain products with a wide range of values we have to normalise the counteroffer. For this purpose, we considered the counteroffer $a_{n}$ in relation to the provisional acquisition price $a_{j}$. We analysed two variants: (i) the ratio of the counteroffer $a_{n}$ to the provisional price $a_{j}$, which we denote by $\rho$ and interpret as the relative price difference; and (ii) the absolute price difference $(\alpha)$ between the counteroffer and the provisional price, i.e.

$$
\begin{aligned}
\rho & :=\frac{a_{n}}{a_{j}}, \\
\alpha & :=a_{j}-a_{n} .
\end{aligned}
$$

Using these two predictor variables in the logistic regression leads to the following probability function for the product holder's acceptance decision:

$$
P(\rho, \alpha)=\operatorname{Pr}[Y=1]=\frac{\exp \left[\beta_{0}+\beta_{1} \rho+\beta_{2} \alpha\right]}{1+\exp \left[\beta_{0}+\beta_{1} \rho+\beta_{2} \alpha\right]} .
$$

Furthermore, we compared the model prediction quality with the cases using only one of the predictor variables. The probability functions for these cases are defined analogously as:

$$
\begin{gathered}
P(\rho)=\operatorname{Pr}[Y=1]=\frac{\exp \left[\beta_{0}+\beta_{1} \rho\right]}{1+\exp \left[\beta_{0}+\beta_{1} \rho\right]}, \\
P(\alpha)=\operatorname{Pr}[Y=1]=\frac{\exp \left[\beta_{0}+\beta_{1} \alpha\right]}{1+\exp \left[\beta_{0}+\beta_{1} \alpha\right]} .
\end{gathered}
$$

We carried out a binary logistic regression analysis for each product category and for all three combinations of predictor variables. The quality of the predictions is summarized in Table 3 . We see that using two 


\begin{tabular}{l|lll} 
& $\rho$ & $\alpha$ & $\rho$ and $\alpha$ \\
\hline All & $79.3 \%$ & $66.3 \%$ & $79.4 \%$ \\
Macs & $83.3 \%$ & $82.3 \%$ & $83.3 \%$ \\
Table & $77.5 \%$ & $72.2 \%$ & $77.5 \%$ \\
NoteNet & $80.8 \%$ & $77.1 \%$ & $81.0 \%$ \\
Phones & $79.6 \%$ & $64.9 \%$ & $79.9 \%$ \\
Cameras & $83.9 \%$ & $79.3 \%$ & $84.5 \%$ \\
MacBooks & $83.2 \%$ & $81.6 \%$ & $83.9 \%$
\end{tabular}

Table 3: Percentage of correct predictions dependent on predictor variable combination for each product category

predictor variables for the complete data set (without differentiating product categories) leads in all to $79.4 \%$ correct predictions with regard to the acceptance decision of the individual product holder. However, the results show that a given change of one unit in the predictor variable $\alpha$ has nearly no influence on the dependent variable $Y \sqrt{2}$ Furthermore, comparing the model quality with only one predictor variable (see again Table 3) reveals that there is nearly no change in the prediction quality for the reduced regression model. Regarding, e.g., all product categories aggregated, we achieve $79.4 \%$ correct prediction with $\alpha$ and $\rho$ as predictor variables and $79.3 \%$ when using only $\rho$. In contrast, the prediction quality drops to $66.3 \%$ when using only $\alpha$ as a predictor. In conclusion, we removed the absolute price difference $\alpha$ from the regression analysis and focused only on the relative price difference $\rho$ as predictor variable for $Y$. Having only a single predictor variable also improves the analytical tractability. Thus, the product holder's acceptance decision $Y$ is approximated by the logistic distribution as in Equation (17).

\subsection{Assessment of counteroffer strategies}

\subsubsection{Optimal counteroffer decision}

We rewrite Equation (13), which describes the recommerce provider's expected profit as a function of the counteroffer by inserting the approximated probability function from Equation (17). To this end, we substitute the updated acquisition price of the counteroffer $a_{n}$ by $\rho a_{j}$. That gives us the following expected profit function for the recommerce provider:

$$
\begin{aligned}
E\left[\Pi^{c}(\rho)\right] & =P(\rho)\left(\Delta m-\rho a_{j}-c-t\right)+(1-P(\rho))(-c-2 t) \\
& =\frac{\Delta m-\rho a_{j}-c-t}{1+e^{-\beta_{0}-\beta_{1} \rho}}+\left(1-\frac{1}{1+e^{-\beta_{0}-\beta_{1} \rho}}\right)(-c-2 t) .
\end{aligned}
$$

\footnotetext{
${ }^{2}$ The odds ratio for $\alpha$ is close to one (e.g. for all product categories $\left.\exp (B)=0.999\right)$ for most product categories. This means that the absolute price difference has only a weak predictive influence on the dependent variable $Y$.
} 
Using this equation, we can determine the optimal counteroffer $\rho^{*}$ which maximizes the recommerce provider's expected profit.

Proposition 1. The unique optimal counteroffer of the recommerce provider is

$$
\rho^{*}:=\min \left(\max \left(\rho^{\prime}, 0\right), 1\right)
$$

with

$$
\rho^{\prime}=\frac{\Delta m+t}{a_{j}}-\frac{1+W\left(e^{\beta_{1}(\Delta m+t) / a_{j}+\beta_{0}-1}\right)}{\beta_{1}}
$$

and $W(\cdot)$ denoting the Lambert $W$ function.

We observe from Proposition 11 that the optimal counteroffer is dependent on the discounted achievable margin $\Delta m$, the provisional acquisition price $a_{j}$, and the transportation costs $t$. Furthermore, the individual behaviour of the product holder is approximated by $\beta_{0}$ and $\beta_{1}$. The grading $\operatorname{costs} c$ have no influence on the optimal solution as they occur in both payoffs, i.e. they are sunk.

Comparing the above optimal counteroffer decision with the results in Section 5.1 .2 of the case of complete information yields insights into the impact of the uncertain residual value on the recommerce provider's acceptance decision. In Section 5.1.2, we observed that the recommerce provider's knowledge of $\delta u$ gives her complete control over the possible outcomes in the acquisition process, once the used product is handedin. Therefore the optimal counteroffer is constant and equal to $a_{n}(\delta u)$ (or simply $\delta u$ when ignoring the discretization effect of the price menu), as long as this value is below $\Delta m+t$, and arbitrarily small otherwise (see (1)). Using the definition of $\rho$ as $\frac{a_{n}}{a_{j}}$, we can rewrite Equation 21 as

$$
a_{n}=\Delta m+t-\frac{\left(1+W\left(e^{\beta_{1}(\Delta m+t) / a_{j}+\beta_{0}-1}\right)\right) a_{j}}{\beta_{1}} .
$$

Equation 222 shows that the optimal counteroffer under uncertainty is also smaller than $\Delta m+t$. Specifically, the recommerce provider undercuts this threshold by a term that reflects the estimated product holder behaviour. Note that $a_{n}$ in 22 is no longer constant but depends on $\Delta m+t$. This reflects the fact that the recommerce provider can no longer predict with certainty whether a given counteroffer will be accepted or not and therefore has to take the payoffs of both cases into account. The optimal counteroffer balances the risk of a rejection with the achievable additional gain of a price reduction.

Analogous with Section 5.1 .2 comparing the expected profit of an optimal counteroffer $E\left[\Pi^{c}\left(\rho^{*}\right)\right]$ with the profit of accepting the provisional acquisition price $\Pi^{a}$ implies whether or not to issue a counteroffer. 


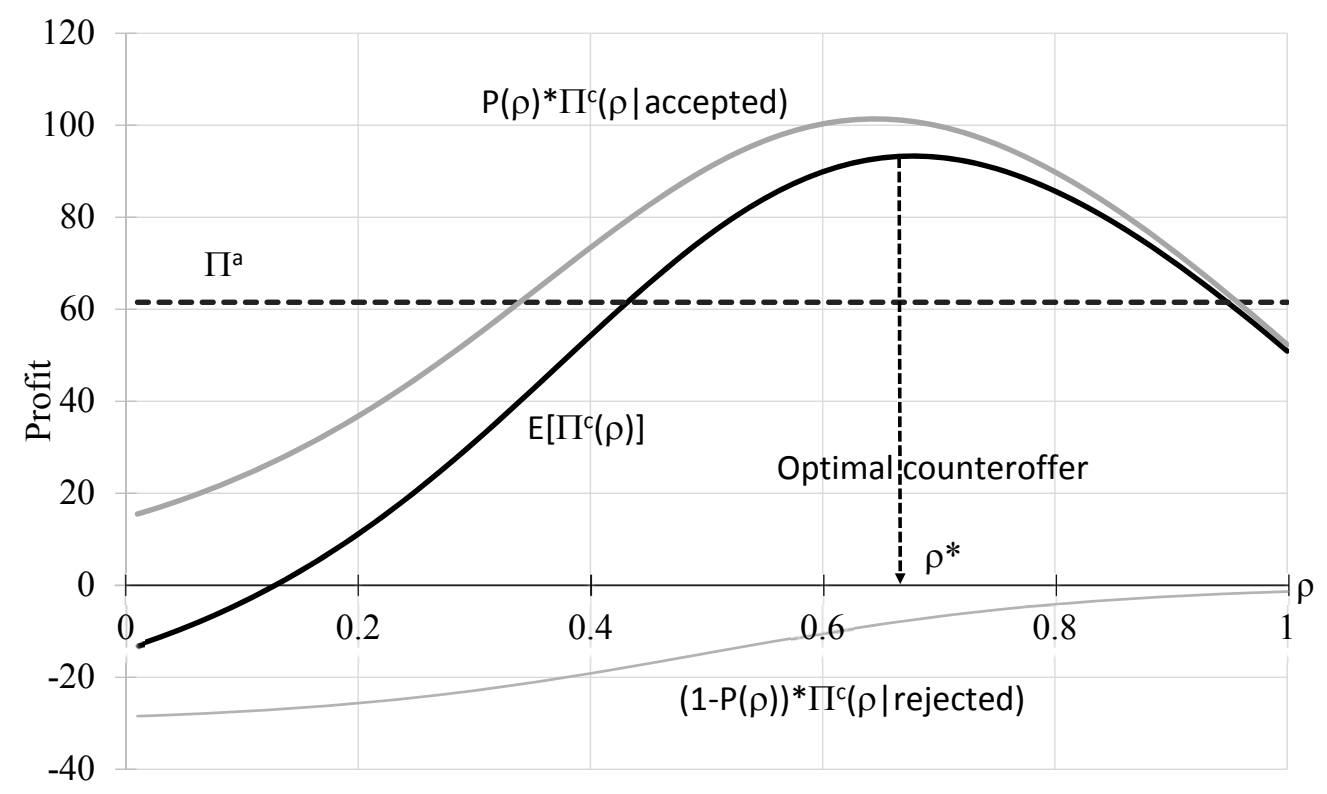

Figure 5: Expected profit for a counteroffer $\left(E\left[\Pi^{c}(\rho)\right]\right)$, profit for direct acceptance of $a_{j}\left(\Pi^{a}\right)$, and weighted payoffs for the counteroffer acceptance and rejection for an exemplary product submission

Inserting 21 into 19 and letting $W:=W\left(e^{\beta_{1}(\Delta m+t) / a_{j}+\beta_{0}-1}\right)$ we obtain $E\left[\Pi^{c}\left(\rho^{*}\right)\right]=\frac{a_{j}}{\beta_{1}} W-c-2 t$. We can then summarize the recommerce provider's optimal strategy as follows. If

$$
\begin{array}{rlrl}
E\left[\Pi^{c}\left(\rho^{*}\right)\right] & \leq \Pi^{a} \\
\Leftrightarrow & \frac{a_{j}}{\beta_{1}} W-c-2 t & \leq m-a_{j}-c-t \\
\Leftrightarrow & a_{j} & \leq \frac{\beta_{1}}{W+\beta_{1}}(m+t)
\end{array}
$$

it is optimal for the recommerce provider to accept the provisional acquisition price $a_{j}$. Otherwise it is optimal to make the counteroffer $\rho^{*}$. Figure 5 illustrates this relationship for a specific product submission. Additionally, this figure shows the probability-weighted payoffs for the counteroffer acceptance and rejection cases, which sum up to $E\left[\Pi^{c}\left(\rho^{*}\right)\right]$.

\subsubsection{Improvement potential of optimized counteroffer strategies}

As discussed in Section 2 FLIP $4 N E W$ currently applies a goodwill rule to decide on a potential counteroffer in the case of a mismatching quality statement. It only makes a counteroffer if the correct quality assessment reveals a price difference which is above a certain threshold. According to FLIP $4 N E W$, the counteroffer equals the price for the true quality $r$ observed in the grading process. The profit functions in Figure 5 support the rationale of this strategy. We observe that for a counteroffer that makes a very small adjustment to 

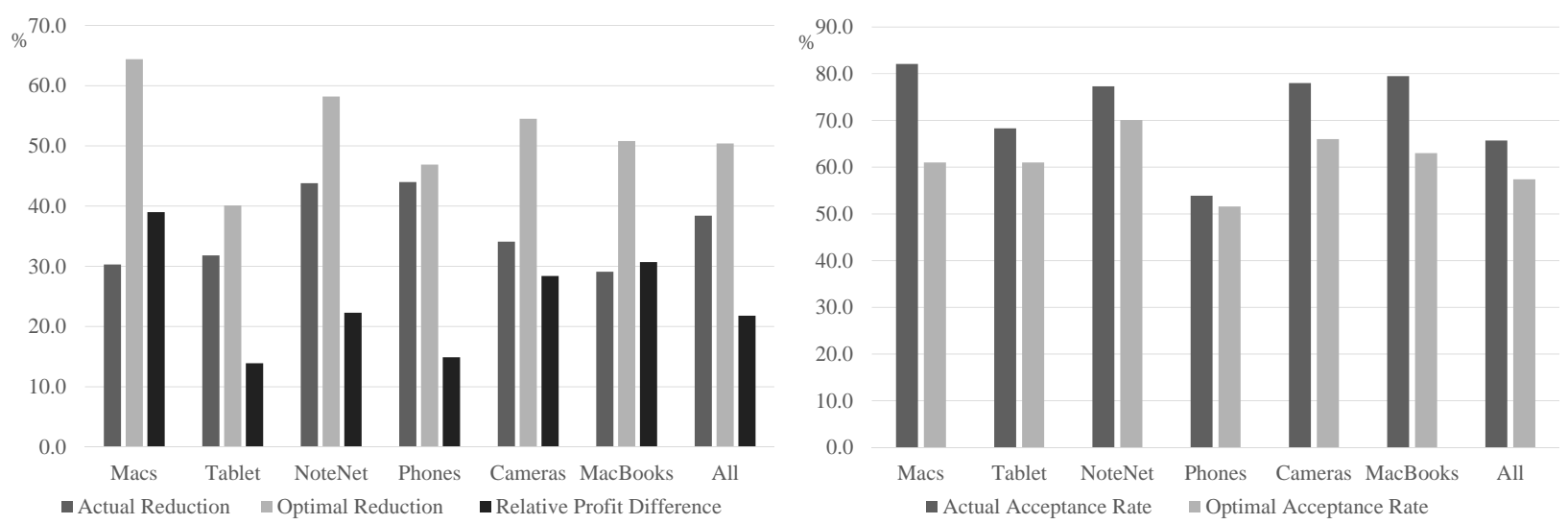

Figure 6: Comparison of optimal counteroffer strategy with the actual applied strategy and the resulting profit difference

\begin{tabular}{l|llllll|l} 
Product Category & Macs & Tablet & NoteNet & Phones & Cameras & MacBooks & All \\
\hline Actual Reduction & 30.3 & 31.8 & 43.8 & 44.0 & 34.1 & 29.1 & 38.4 \\
Optimal Reduction & 64.4 & 40.1 & 58.2 & 46.9 & 54.5 & 50.8 & 50.4 \\
Actual Accept. Rate & 82.1 & 68.3 & 77.3 & 53.9 & 78.0 & 79.5 & 65.7 \\
Optimal Accept. Rate & 61.0 & 61.0 & 70.1 & 51.6 & 66.0 & 63.0 & 57.4 \\
Relative Profit Diff. & 39.0 & 13.9 & 22.3 & 14.9 & 28.4 & 30.7 & 21.8
\end{tabular}

Table 4: Comparison of optimal counteroffer strategy with the actual applied strategy and the resulting profit difference (in percent)

the acquisition price the potential additional gain does not offset the risk of a rejection of the counteroffer. Thus, for a product submission that only slightly deviates from the stated quality, it is more beneficial for FLIP $4 N E W$ to accept the inaccurate provisional acquisition price.

In the following, we compare the current counteroffers in the available data set with the optimized counteroffers according to our logistic regression model. To this end, we determined the optimal counteroffer $\left(\rho^{*}\right)$ for each product submission ${ }^{3}$ based on Proposition 1 and compared it with the actual counteroffer by FLIP $4 N E W$. We restrict our analysis to the product submissions for which FLIP $4 N E W$ issued a counteroffer since the underlying estimation of the product holder behaviour is based on these submissions. Figure 6 and Table 4 summarize this comparison by illustrating the average counteroffers of both strategies for each product category, together with their respective counteroffer acceptance rates. Additionally, we state the average relative profit difference between the two strategies according to Equation (19).

The comparison reveals several interesting points. First, the optimal counteroffer $\rho^{*}$ calculated in the model is significantly lower than that of the actual strategy. The estimation of the product holder behaviour suggests lower counteroffers to optimally balance the trade-off between higher gains and lost acquisitions. Thus, it appears FLIP 4 NEW's current goodwill rule of only issuing counteroffers in the case of a certain

\footnotetext{
${ }^{3}$ The exact data of the achieved margin $(\Delta m)$ is only available for the accepted counteroffers, as these products are actually resold. For the rejected counteroffers, we have approximated the achievable margin by the average margin for this product category.
} 
minimum price deviation still results in an insufficient price reduction, relative to the associated rejection probability. One possible explanation for the observed deviation is that FLIP $4 N E W$ does not only have a short-term view of individual product submission, as our model assumes, but is interested in achieving long-term customer loyalty for generating profits from future product submissions. Thus, if a counteroffer rejection means that a product holder will not use the services of the recommerce provider in the future, the resulting losses of the rejection are higher than the immediate losses we consider in our model. Another possible explanation for the observed deviation is that FLIP $4 N E W$ restricts itself for most product categories to counteroffers which are defined by the discrete predefined price vector $a_{i}$ whereas the developed model selects the optimal counteroffer $\rho^{*}$ in the interval $[0,1]$.

Additionally, the results show that the optimal counteroffer $\rho^{*}$ is dependent on the product categories. Interestingly, the categories with the highest optimal counteroffer $\rho^{*}$ contain bulkier products (Macs: $64.4 \%$, NoteNet: $58.2 \%$ ) while for the categories which contain smaller products (Phones: 46.9\%, Tablets: 40.1\%) the optimal counteroffer is lower. One possible explanation for this is that the bulkiness of a returned product has an influence on the individual acceptance decision of a product holder faced with a counteroffer, which is in line with our interpretation of the discount factor $\delta$. Since the handling effort for taking back bulkier products is greater, the product holders are willing to accept a larger price decrease. We already addressed this impact of $\delta$ on the profit allocation between the players in the analysis of the equilibrium solution in Section 5.2. It is also in line with other research concerning the bulkiness of the returned products Wojanowski et al. 2007, Aras and Aksen, 2008, Hahler and Fleischmann, 2013).

The data set also shows that the product category Phones accounts for the largest volume, i.e. $60 \%$ of the total returns. Interestingly, for this product category, the differences between the currently applied and the optimal counteroffer in Figure 6 is only 2.9\%. This is the lowest value of all product categories. This suggests that the current policy is quite appropriate for the company's most important product category. Nevertheless, the estimated profit difference between the two strategies of $14.9 \%$ is still significant.

Overall, we observe that the profit difference for individual product categories amounts to up to $39 \%$, and is $21 \%$ aggregated for all product categories. This shows how relevant the counteroffer decision is for the profitability of a recommerce provider and it suggests potential for improvement. Critical points that we must consider with regard to these results are the aforementioned limitations of our model approach with respect to neglecting the long-term effects on customer loyalty and a possible misalignment of the optimal continuous counteroffer $\rho^{*}$ with the discrete price vector $a_{i}$. Furthermore, we have to take into account the achieved prediction quality of the product holder behaviour which is about $80 \%$. 


\section{Conclusions}

Our research is motivated by a close collaboration with a recommerce provider, during which we observed newly emerging issues in the assessment of used products. We propose a basic model which describes the acquisition process between a recommerce provider and a product holder in a sequential game. We derive the optimal strategies of both players under complete information and observe that the recommerce provider can achieve most of the potential profit due to her last mover advantage. Furthermore, the equilibrium of the game illustrates the existing incentive problem between the players, concerning the correct assessment of the used products. By modifying this basic model, we evaluate levers like shifting transportation costs and additional payments for correct quality statements, and determine their effect on the optimal strategies of both players. In the case of a transportation cost shift, the recommerce provider gains even more bargaining power as the payoffs of the last period are reduced for the product holder. A bonus payment for correct grading has no effect on the optimal strategies of the players.

We extend the basic model and incorporate uncertainty about the product holder's residual product valuation. A data set consisting of nearly 60,000 product acquisitions of a recommerce provider is used to estimate the individual product holder's return behaviour empirically. Specifically, we develop a logistic regression approach to approximate the counteroffer acceptance decision of the product holder, dependent on the relative price decrease of the counteroffer. For this extended counteroffer decision model, we derive the optimal product assessment strategy of the recommerce provider. A comparison with the currently applied counteroffer strategy of the recommerce provider suggests significant potential of our newly developed strategy, under the given assumptions. Furthermore, we observed the influence of the product category on the individual product holder's return behaviour. We identified the bulkiness of a product as a significant factor.

Our main contributions to the literature are the introduction of the recommerce business to the field of reverse logistics, the identification of the recently implemented acquisition process, and the exploration of the emerging incentive problem when assessing the used products.

We see opportunities for future research in relaxing some of our modeling assumptions in our approach. We think that a relaxation of the assumption of complete information in the sequential game can lead to further important results concerning the incentive problem. Furthermore, our present derivation of the optimal strategies does not consider long-term effects on the behaviour of the product holder. In particular, if all handed-in products of product holders were to be downgraded independently of the stated quality class, this would lead to a dissatisfied customer base. Additionally, we believe that the recommerce provider's decision about the initial price vector entails an interesting opportunity for future research. 


\section{Appendix}

\section{Proof of Proposition 1 .}

The first order derivative of Equation $(19)$ is

$$
\partial E[\Pi(\rho)] / \partial \rho=\frac{\left(\left(-1-\beta_{1} \rho\right) a_{j}+(\Delta m+t) \beta_{1}\right) e^{-\beta_{0}-\beta_{1} \rho}-a_{j}}{\left(1+e^{-\beta_{0}-\beta_{1} \rho}\right)^{2}}
$$

and the first-order condition $\partial E[\Pi(\rho)] / \partial \rho=0$ results in

$$
\rho^{\prime}=\frac{\Delta m+t}{a_{j}}-\frac{1+W\left(e^{\beta_{1}(\Delta m+t) / a_{j}+\beta_{0}-1}\right)}{\beta_{1}}
$$

with $W(\cdot)$ denoting the Lambert $W$ function. As $e^{(\cdot)}>0$, the Lambert $W$ function is unique, and thus $\rho^{\prime}$ is also unique.

Considering Equation 23 , we observe that $\left(-1-\beta_{1} \rho\right) a j+(\Delta m+t) \beta_{1}$ and $e^{-\beta_{0}-\beta_{1} \rho}$ are both decreasing in $\rho$ as $\beta_{1}$ is always positive in our setting Since furthermore, $e^{-\beta_{0}-\beta_{1} \rho}>0$, it follows that $\partial E[\Pi(\rho)] / \partial \rho$ is positive for $\rho<\rho^{\prime}$ and negative for $\rho>\rho^{\prime}$. Thus, Equation (19) is unimodal with its maximum value at $\rho^{\prime}$. As the counteroffer decision is only defined for $\rho$ in the interval $[0,1]$, the optimal counteroffer $\rho^{*}$ is $\rho^{\prime}$ in case $0 \leq \rho^{\prime} \leq 1$. Otherwise, due to the unimodality of Equation $\left.\sqrt{19}\right)$, the optimal counteroffer $\rho^{*}$ is $1(0)$ for the case that $\rho^{\prime}>1\left(\rho^{\prime}<0\right)$.

\footnotetext{
${ }^{4}$ The ratio of the counteroffer to the provisional acquisition price $\left(\rho=a_{n} / a_{j}\right)$ is positively correlated with the counteroffer acceptance decision of the product holder.
} 


\section{References}

Aras, N. and D. Aksen (2008). Locating collection centers for distance- and incentive-dependent returns. International Journal of Production Economics 111(2), 316-333.

Aras, N., T. Boyaci, and V. Verter (2004). The effect of categorizing returned products in remanufacturing. IIE Transactions 36(4), 319-331.

Blackburn, J. D., V. D. R. Guide, G. C. Souza, and L. N. Van Wassenhove (2004). Reverse supply chains for commercial returns. California Management Review 46(2), 6-22.

European Commission (2013). http://ec.europa.eu/justice/consumer-marketing/rights-contracts/directive/.

Fleischmann, M., M. R. Galbreth, and G. Tagaras (2010). Product aquisition, grading, and disposition decisions. In M. E. Ferguson and G. Souza (Eds.), Closed-Loop Supply Chains - New Developments to Improve the Sustainability of Business Practices, Chapter 6, pp. 100 - 118. Auerbach Publishers Inc.

Fudenberg, D. and J. Tirole (1991). Game theory. 1991.

Gönsch, J. (2014). Buying used products for remanufacturing: negotiating or posted pricing. Journal of Business Economics 84(5), 715-747.

Guide, V. D. R. and V. Jayaraman (2000). Product acquisition management: current industry practice and a proposed framework. International Journal of Production Research 38(16), 3779-3800.

Guide, V. D. R., L. Muyldermans, and L. N. Van Wassenhove (2005). Hewlett-packard company unlocks the value potential from time-sensitive returns. Interfaces 35(4), 281-293.

Guide, V. D. R., G. C. Souza, L. N. Van Wassenhove, and J. D. Blackburn (2006). Time value of commercial product returns. Management Science 52(8), 1200-1214.

Guide, V. D. R., R. H. Teunter, and L. N. Van Wassenhove (2003). Matching demand and supply to maximize profits from remanufacturing. Manufacturing 83 Service Operations Management 5(4), 303-316.

Guide, V. D. R. and L. N. V. Wassenhove (2001). Managing product returns for remanufacturing. Production and Operations Management 10(2), 142-155.

Guide, V. D. R. and L. N. V. Wassenhove (2009). The evolution of closed-loop supply chain research. Operations Research 57(1), 10-18.

Hahler, S. and M. Fleischmann (2013). The value of acquisition price differentiation in reverse logistics. Journal of Business Economics 83(1), 1-28. 
Hosmer Jr, D. W., S. Lemeshow, and R. X. Sturdivant (2013). Applied logistic regression. Wiley. com.

Karakayali, I., H. Emir-Farinas, and E. Akcali (2007). An analysis of decentralized collection and processing of end-of-life products. Journal of Operations Management 25(6), 1161-1183.

Ketzenberg, M. E., G. C. Souza, and V. D. R. Guide (2003). Mixed assembly and disassembly operations for remanufacturing. Production and Operations Management 12(3), 320-335.

Ray, S., T. Boyaci, and N. Aras (2005). Optimal prices and trade-in rebates for durable, remanufacturable products. Manufacturing \& Service Operations Management 7(3), 208-228.

Souza, G. C. (2013). Closed-loop supply chains: A critical review, and future research. Decision Sciences $44(1), 7-38$.

Souza, G. C., M. E. Ketzenberg, and V. D. R. Guide (2002). Capacitated remanufacturing with service level constraints. Production and Operations Management 11(2), 231-248.

Taube, H. (2013). Incentive problems in the quality grading of used products. Master's thesis, University of Mannheim.

Wojanowski, R., V. Verter, and T. Boyaci (2007). Retail-collection network design under deposit-refund. Computers 8 Operations Research 34(2), $324-345$.

Zikopoulos, C. and G. Tagaras (2008). On the attractiveness of sorting before disassembly in remanufacturing. IIE Transactions 40(3), 313-323. 